\title{
A SPECIFIC PROBLEM OF MECHANISM SYNTHESIS
}

\author{
J. BUŚKIEWICZ \\ Division of Technical Mechanics \\ Faculty of Mechanical Engineering and Management \\ Poznan University of Technology \\ Jana Pawła II str. 24 \\ 60-965 Poznań, POLAND \\ E-mail: jacek.buskiewicz@put.poznan.pl
}

\begin{abstract}
A technique for path synthesis is employed to design a feeder for carrying products between two points. The feeder is assumed to be a one degree of freedom system of six links connected by means of revolute joints. The mathematical basis of the concept is presented. An exemplary solution is presented and discussed.
\end{abstract}

Key words: six-bar linkage, path synthesis, feeder design.

\section{Introduction}

In many machines the motion of two floating links has to be coupled in the working phase. Very special techniques for determining the structure and dimensions of such mechanisms must be employed. In such cases the most useful are methods worked out for typical synthesis tasks, which can be adopted to solve special synthesis tasks. A feeder for carrying a load between two points is considered in this paper. The feeder is assumed to be a one degree of freedom system of six links connected by means of revolute joints. In the 1-DOF feeder the motions of two links suspending jaws of the gripper have to be mechanically coupled, and these links are driven by one active link. When a product is transported by the feeder there is no relative motion between these links - the gripper is closed. It means that the phase of motion exists when two links rotate at the same angular velocity. Zero relative angular velocity is the equation coupling motions of these links. The jaws open when the product is released. A concept of employing a path/motion synthesis method in designing such a feeder was introduced in Buśkiewicz (2014). The aim of the paper is to propose another method for solving this problem. As in Buśkiewicz (2014), the method is also derived from the technique worked out for the optimal path/motion synthesis without time prescription of four link planar mechanisms (Buśkiewicz, 2009). The method enables decreasing the number of design parameters describing dimensions, orientation and position of a path/motion generator. As the mechanism synthesis is a problem of great mathematical complexity (Suh and Radcliffe, 1978; Erdman et al., 2001), at present mainly computer methods are developed. The effectiveness of the method depends essentially on the number of design parameters, therefore mechanism synthesis addresses also the techniques minimizing the number of design parameters (Buśkiewicz, 2014; Buśkiewicz, 2010; McGarva and Mullineux, 1994; Ullah and Kota, 1997; Wen-Yi, 2010). Other techniques focus on developing algorithms minimizing an objective function and constructing new error functions (Penunuri et al., 2011; Sanchez Marin and Gonzalez, 2004; Matekar and Gogate, 2012; Bulatovic and Dordevic, 2009; Chao and Jorge, 2008). 


\section{Review on the carrier machines}

The industrial robots are the state-of-the-art solutions in the processes of self-operating transport between production zones. The robots are multi-degree of freedom solutions. In feeders equipped with hydraulic systems the gripper requires the drive independent of the other feeder links. The simplest solution is one degree of freedom kinematic systems built of as small as possible number of links. Grippers are known which fail to require an additional, independent mechanical drive or an electronic steering. In these feeders a load is pressed by the arm attached to the pull spring in the phase of carrying (Patent, P.102470; P.125376; P.111933). The feeders based on the mechanisms in which no pull strings are applied are also presented (Suh and Radcliffe, 1978; Erdman et al., 2001; Hain, 1967; Gronowicz et al., 1991).

The motivation of the paper is to design a feeder in which the jaws of the gripper are open when the load is caught, they close, and remain closed when the load is being transported and open when the load is taken away, besides the motion is transmitted mechanically from the driving link to the jaws, which do not require any additional independent control, steering and other tightening elements.

\section{The geometry of the feeder driven by a four-bar linkage}

\subsection{Description of the synthesis method}

A four bar linkage is the basic kinematic chain of the feeder. The path synthesis method adapted to design the feeder is briefly presented (Buśkiewicz, 2009).

We synthesize the four-bar linkage $\mathrm{O}_{1} \mathrm{ABO}_{2}$ a point which traces the trajectory. Let us disconnect the coupler from the four-bar linkage as shown in Fig.1. We assume that the coupler point D traces exactly a required open path, given parametrically

$$
x=x(s), \quad y=y(s)
$$

where $0 \leq s<1$. Simultaneously the coupler rotates according to the function

$$
\theta_{2 L}=v_{1} \sin \left(v_{2} s+v_{3}\right)+v_{4} .
$$

The aim is to specify the locations of joints $\mathrm{A}$ and $\mathrm{B}$ to connect the coupler with the rotating input and output links. In the ideal case these joints move on circular arcs. Let us denote: $|\mathrm{AD}|=l_{5},|\mathrm{BD}|=l_{6}, \quad$ is the angle between segments DA and DB. Hence, to solve the synthesis problem for the four bar linkage as a path generator one has to determine:

- the lengths of the coupler $l_{5}, l_{6}$, and angle $\beta$,

- the coefficients of function (3.2): $v_{1}, v_{2}, v_{3}, v_{4}$,

so that the revolute joints A and B of the coupler would trace as close as possible the trajectories which they trace in the assembled mechanism when point $\mathrm{D}$ traces the required trajectory $(2.1)$. 


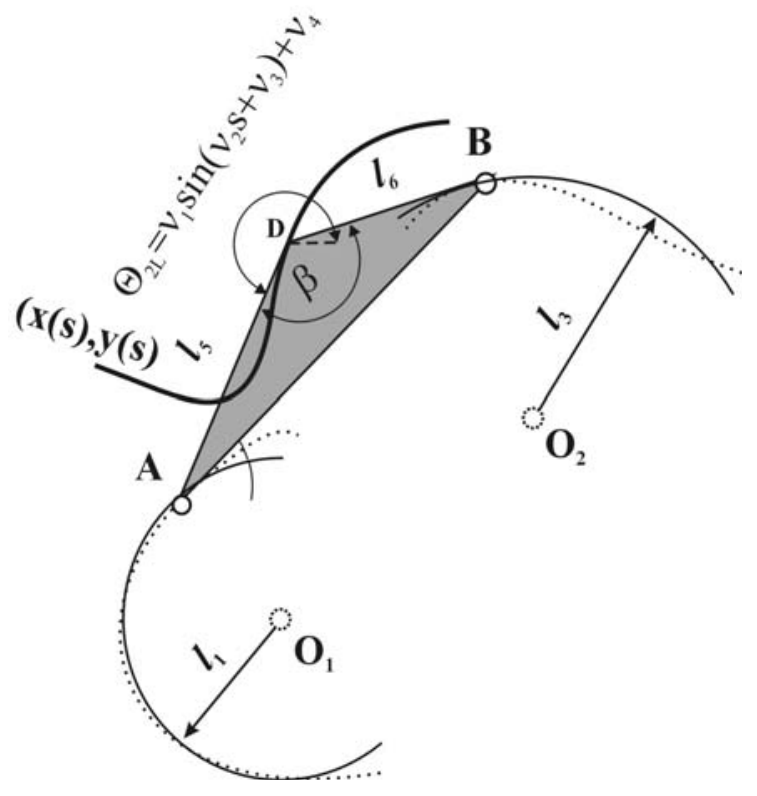

Fig.1. Computed paths of joints A and B and circular arcs approximating these paths.

Seven parameters have to be determined. In the paper (Buśkiewicz, 2009) it was proved that the angular motion of the coupler can be expressed by means of sine function (3.2). The method belongs to the group of methods aimed at decreasing the number of optimized design parameters. In general, the geometry of the four bar linkage as an open path generator is defined by 11 parameters including link lengths, coordinates of ground pins, location of a coupler point and the boundaries of the angle through which the active link rotates when the path is traced.

The design parameters are optimized by means of an evolutionary algorithm. The algorithm itself involves no special improvements. The objective function measures the deviation of joints $\mathrm{A}$ and $\mathrm{B}$ from ideal circles. Assume that the path of joint $\mathrm{A}$ is discretized into $m=20$ points. We determine the circles passing through three points $\mathrm{A}_{i}, \mathrm{~A}_{5+i}$ and $\mathrm{A}_{10+i}, i=1 . .10$. Then, we obtain 10 circles. We compute the centroid of their centres and the average value of their radiuses. In this manner we obtain the position of joint $\mathrm{O}_{l}$ and the length of the active link $l_{l}$. The same procedure enables determination of the coordinates of the output link ground pin $\mathrm{O}_{2}$ and the output link length $l_{3}=\left|\mathrm{O}_{2} \mathrm{~B}\right|$. The objective function has the form

$$
E=\frac{\max \left|\mathrm{O}_{1} \mathrm{~A}_{i}\right|-\min \left|\mathrm{O}_{1} \mathrm{~A}_{i}\right|}{l_{l}}+\frac{\max \left|\mathrm{O}_{2} \mathrm{~B}_{i}\right|-\min \left|\mathrm{O}_{2} \mathrm{~B}_{i}\right|}{l_{3}} . i=1 . . m .
$$

One can also prescribe the locations of the ground pins. Then, the objective function is a measure of deviation of joints $\mathrm{A}$ and $\mathrm{B}$ trajectories from the circles with centres at prescribed points $\mathrm{O}_{1}$ and $\mathrm{O}_{2}$.

\subsection{Adaptation of the synthesis method for specific requirements}

A path along which the product is transported is given. We disconnect the coupler from the rotating links. Point $\mathrm{D}$ of the coupler moves along the prescribed path. The dimensions of the coupler and the parameters of the function (3.2) are such that three other points of the coupler approximate circular arcs. Two of them are joints $\mathrm{A}$ and $\mathrm{B}$ connecting the coupler to the rotating links. The third point $\mathrm{N}^{\prime}$ lies on an approximate circular arc of radius $R$ and centred at $\mathrm{O}_{3}$. The geometric scheme of this concept is presented in 
Fig.2a. When point $\mathrm{D}$ traces the required path, point $N^{\prime}$ is coincident with point $N$ lying on an additional link (fixed to the ground by the pivot at $\mathrm{O}_{3}$ ). The length of this link is $l_{7}=R$. Link $\mathrm{O}_{3} \mathrm{~N}$ is connected to coupler $\mathrm{AB}$ by means of an additional link $\mathrm{MN}$ where the position of revolute joint $\mathrm{M}$ on coupler $\mathrm{AB}$ is chosen arbitrarily. The links $\mathrm{MN}$ and $\mathrm{NO}_{3}$ are connected by revolute joint $\mathrm{N}$. In the initial position the coordinates of points $\mathrm{N}^{\prime}$ and $\mathrm{N}$ are equal to each other. The location of point $\mathrm{M}$ has to provide a proper motion of the sixlink mechanism, and must be changed when, e.g., dead position occurs. Joint $\mathrm{N}$ moves along the arc of the circle with centre at point $\mathrm{O}_{3}$ and in the ideal case its position is coincident with the position of point $\mathrm{N}^{\prime}$ when $\mathrm{D}$ moves along the prescribed path. The remaining parts of the paths of joint $\mathrm{N}$ and point $\mathrm{N}^{\prime}$ differ from each other. Then, we designed a six-link planar mechanism (Radovan et al., 2013; Kramer and Sandor, 1970). A distinguishing feature of its own is a phase of motion in which links $\mathrm{AB}$ and $\mathrm{MN}$ do not rotate with respect to each other (Fig.2b). The scheme of the feeder with jaws $\mathrm{D}_{1}$ and $\mathrm{D}_{2}$ attached to couplers $\mathrm{AB}$ and $\mathrm{MN}$ is presented in Fig. 3 (point $\mathrm{D}$ is replaced by point $\mathrm{D}_{l}$ of the first jaw).

a)

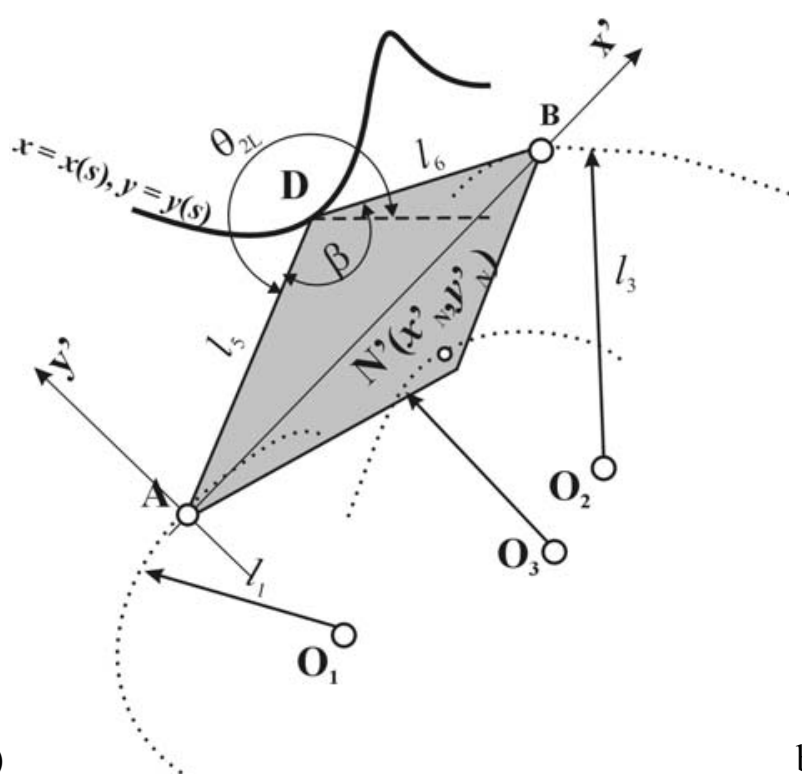

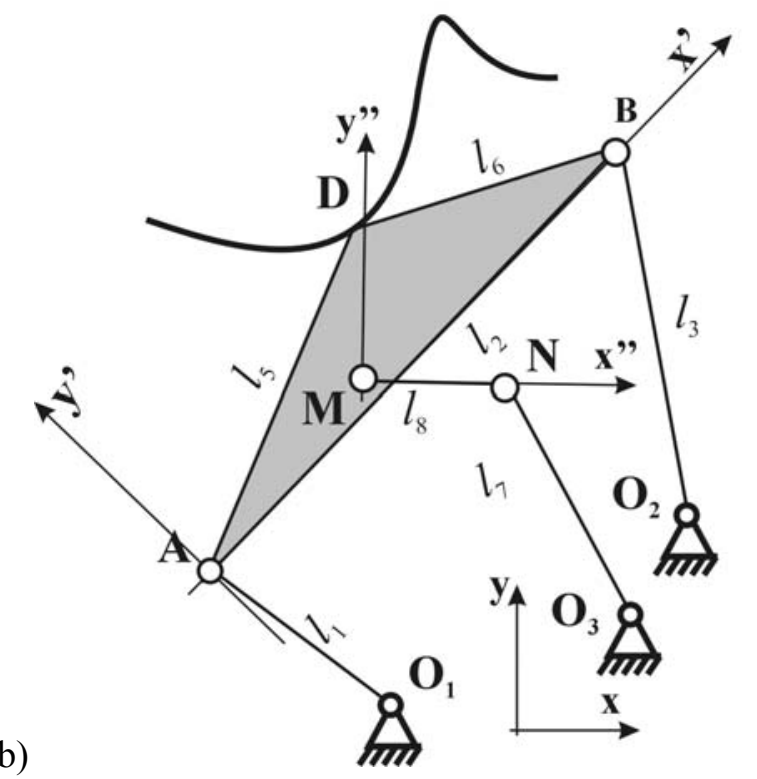

Fig.2. The geometric scheme of the concept (a), the feeder constructed on the basis of determined coupler dimensions (b).

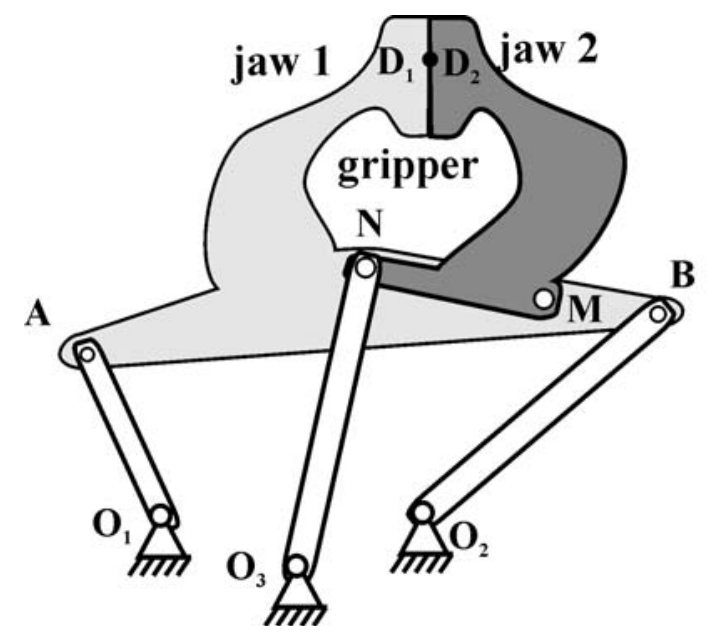

Fig.3. The feeder with the gripper. 
The local system Ax'y' is attached to coupler AB. In the approach presented herein the path synthesis method described in section 3.1 is modified in order to determine:

- the lengths of coupler $\mathrm{AB} l_{5}, l_{6}$, and angle $\beta$,

- the coordinates $x_{N}^{\prime}, y_{N}^{\prime}$ of point $\mathrm{N}^{\prime}$ in the local system $A x^{\prime} \mathrm{y}^{\prime}$,

- the coefficients of function (3.2): $v_{1}, v_{2}, v_{3}, v_{4}$,

so that the revolute joints $\mathrm{A}$ and $\mathrm{B}$ as well as point $\mathrm{N}^{\prime}$ of the coupler would trace as close as possible circular arcs when point $\mathrm{D}$ traces the required trajectory (3.1). Compared with the original version of the synthesis method, the number of design parameter is by two grater.

The modified objective function has the form

$$
E=\frac{\max \left|\mathrm{O}_{1} \mathrm{~A}_{i}\right|-\min \left|\mathrm{O}_{1} \mathrm{~A}_{i}\right|}{l_{1}}+\frac{\max \left|\mathrm{O}_{2} \mathrm{~B}_{i}\right|-\min \left|\mathrm{O}_{2} \mathrm{~B}_{i}\right|}{l_{3}}+\frac{\max \left|\mathrm{O}_{3} \mathrm{~N}_{i}^{\prime}\right|-\min \left|\mathrm{O}_{3} \mathrm{~N}_{i}^{\prime}\right|}{l_{8}} .
$$

The locations of ground pins $\mathrm{O}_{1}, \mathrm{O}_{2}$ and $\mathrm{O}_{3}$ may be prescribed. Given links lengths one can determine angles $\theta_{7}$ and $\theta_{8}$ defining positions of links $\mathrm{O}_{3} \mathrm{~N}$ and $\mathrm{MN}$. The angles are computed from the vector closed loop equation

$$
\mathbf{O}_{3} \mathbf{N}=\mathbf{O}_{2} \mathbf{B}+\mathbf{B M}+\mathbf{B N}
$$

which can be rewritten as two algebraic equations

$$
\begin{aligned}
& l_{7} \cos \theta_{7}+l_{8} \cos \theta_{8}=x_{M}-x_{O 3}, \\
& l_{7} \sin \theta_{7}+l_{8} \sin \theta_{8}=y_{M}-y_{O 3} .
\end{aligned}
$$

This concept differs from the one presented in the paper (Buśkiewicz, 2014) in the way the synthesis problem is formulated. In Buśkiewicz (2014) the circular arc of joint $\mathrm{N}$ is the synthesized path under constraints that joints A and B move along circular arcs. Then, the position of the jaw (point D) is specified manually. In the present concept the path of the jaw is synthesized under constraints that joints $\mathrm{A}, \mathrm{B}$ and $\mathrm{N}$ move along circular arcs.

\section{A numerical solution}

The concept is illustrated with a numerical solution. The four-bar linkage is the basic kinematic chain that transmits motion from the active link to the other links of the feeder. The first step consists in determining the four bar linkage by means of the presented method. The evolutionary algorithm was employed to minimise the objective function (3.4). The following constraints may be given:

- The coordinates of the pivots of rotating links - one can specify the intervals for the locations of these pivots.

- The ranges for links lengths.

- The range for transmission angle variation - it is recommended that the angle between the coupler and the passive rotating link varies between $30^{\circ}$ and $150^{\circ}$. 


\section{Input data:}

The feeder is supposed to meet the following preset conditions expressed nondimensionally:

- coordinates of joint $\mathrm{O}_{I}(-3,0)$,

- coordinates of joint $\mathrm{O}_{2}(x, 0)$, where $0<x<10$,

- the lengths of links have to be less than 10 ,

- the coordinates of joint $\mathrm{O}_{3}(0,0)$.

The feeder transports a product along a straight line starting at point $(7,7.5)$ and ending at $(10.5,11)$. This line is the path of coupler point $\mathrm{D}$.

\section{Solution:} is as follows:

Coupler point D approximates the synthesized straight line. An outcome satisfying the requirements

- coupler dimensions: $l_{5}=15.03, l_{6}=17.48, \beta=0.609 \mathrm{rad}$,

- the coordinates of point $\mathrm{N}^{\prime}$ in the local system attached to coupler AB: $(2.59,-0.78)$

- the coefficients of the angular position of the coupler: $\theta_{2 L}=0.41 \sin (-1.66 s+5.7)+3.61 \mathrm{rad}$.

Further, on the basis of these outputs, we compute:

- the lengths of the rotating active and passive links: $l_{1}=2.67, l_{3}=7.55$,

- the horizontal coordinate of ground pivot $\mathrm{O}_{2}: x_{O 2}=9.85$,

- the length $l_{2}=10$,

- the angle through which the active link rotates when the arc is traced: $0.862<\theta_{1}<3.29 \mathrm{rad}$.

Link $\mathrm{O}_{3} \mathrm{~N}$ is pinned at point $\mathrm{O}_{3}$ and its length $l_{7}=3$.

Now, the coordinates of point $\mathrm{M}$ at which links $\mathrm{NM}$ and $\mathrm{AB}$ are connected should be chosen. To avoid singular positions of links $\mathrm{O}_{3} \mathrm{~N}$ and $\mathrm{NM}$, the coordinates of joint $\mathrm{M}$ in the local coordinate $\mathrm{Ax}$ ' $y$ ' are $(11,1)$. Then, the length of link $\mathrm{MN}$ is $l_{8}=8.59$.

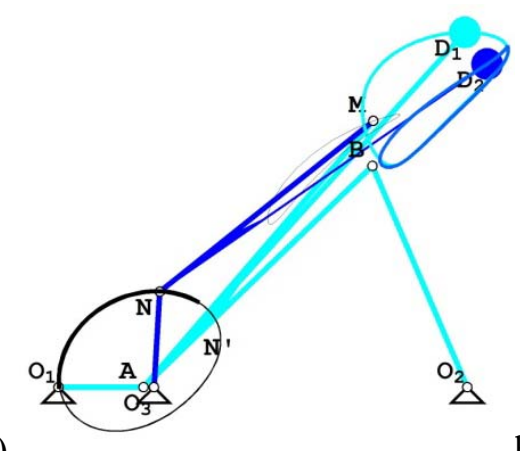

a)

b)

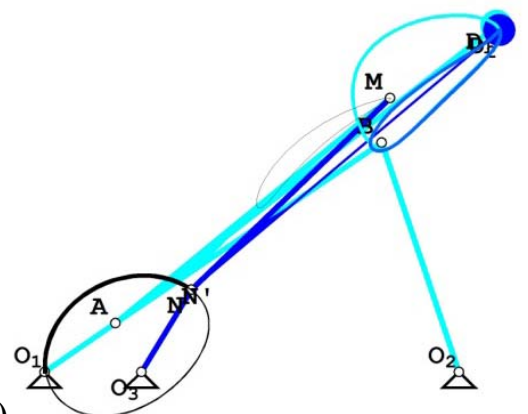

c)

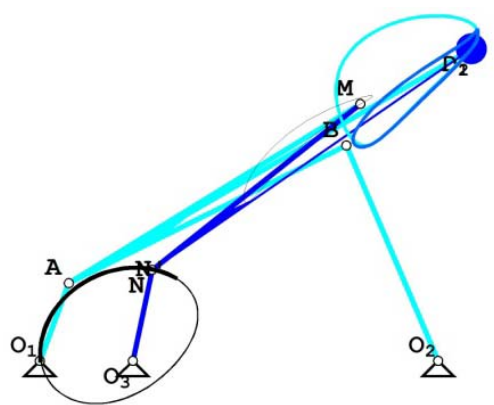


d)

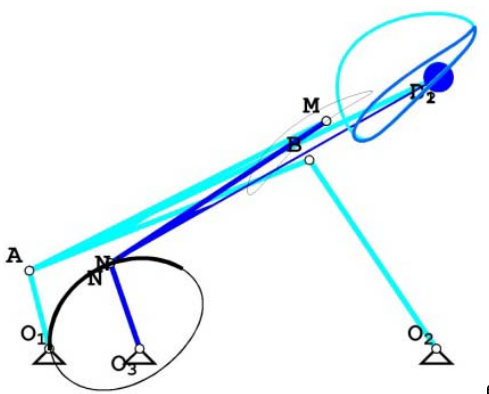

e)

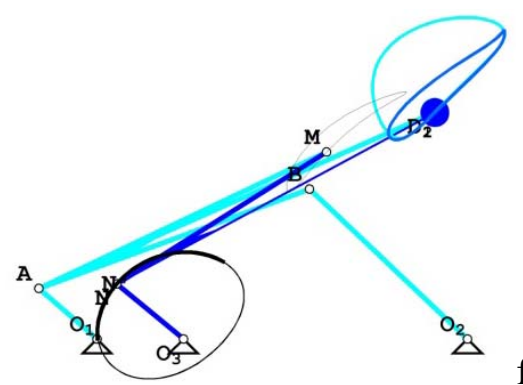

f)

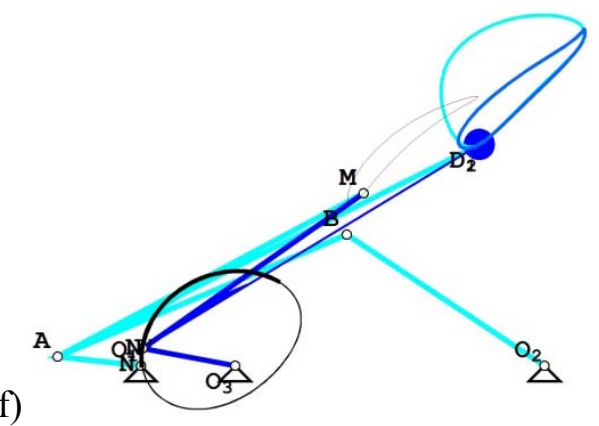

g)

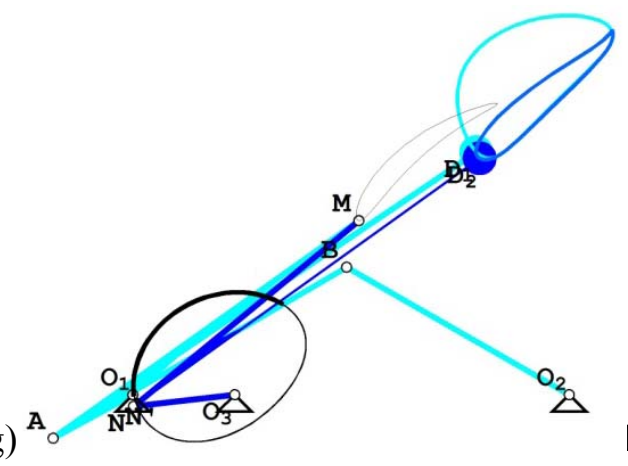

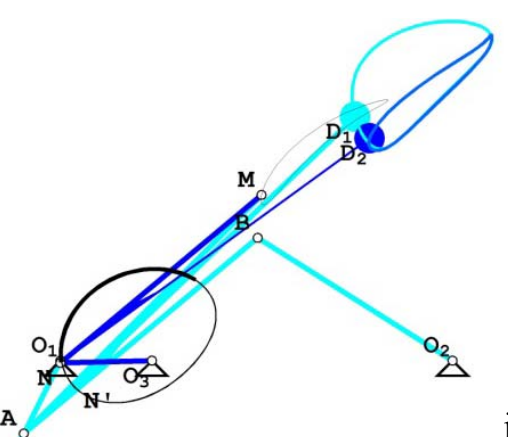

i)

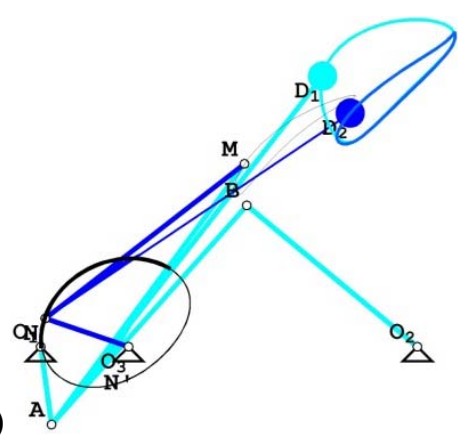

j)

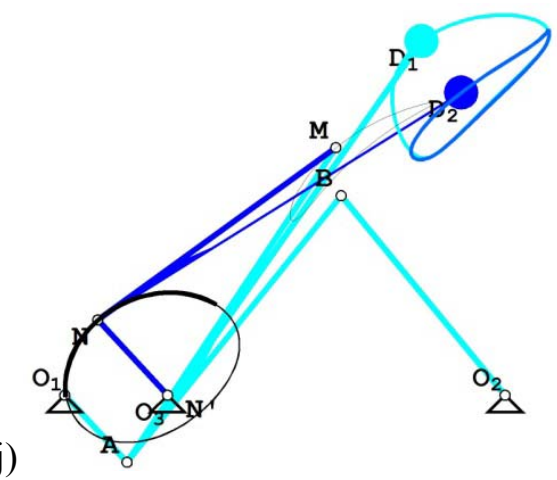

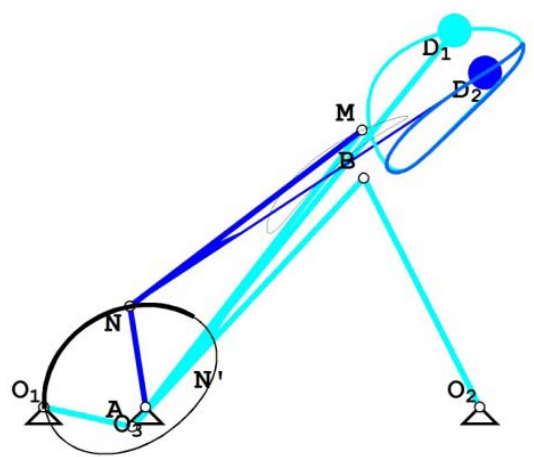

Fig.4. Positions of the mechanism while transporting the load (the trajectories of $\mathrm{D}_{1}$ and $\mathrm{D}_{2}$ overlaps, the gripper is closed and traces straight line) and the return motion.

As the size of the space occupied by the moving feeder is important for a designer, the paths of all joints which influence on the size of the housing are presented. Figures 4a-k show instants when the gripper is closed and open. Point $N^{\prime}$ coincides with joint $N$ when jaws are closed. The paths of both jaws $\mathrm{D}_{1}, \mathrm{D}_{2}$ are given, moreover the path traced by joint $M$ is drawn as it also affects the working space of the feeder.

As shown in Fig.5, the difference between the angular positions of both links supporting the jaws is very small. When the load is transported, the relative rotation of the couplers is less than $0.01 \mathrm{rad}$. The transmission angle of the four-bar linkage $\mathrm{O}_{1} \mathrm{ABO}_{2}$ is presented in Fig.6. 


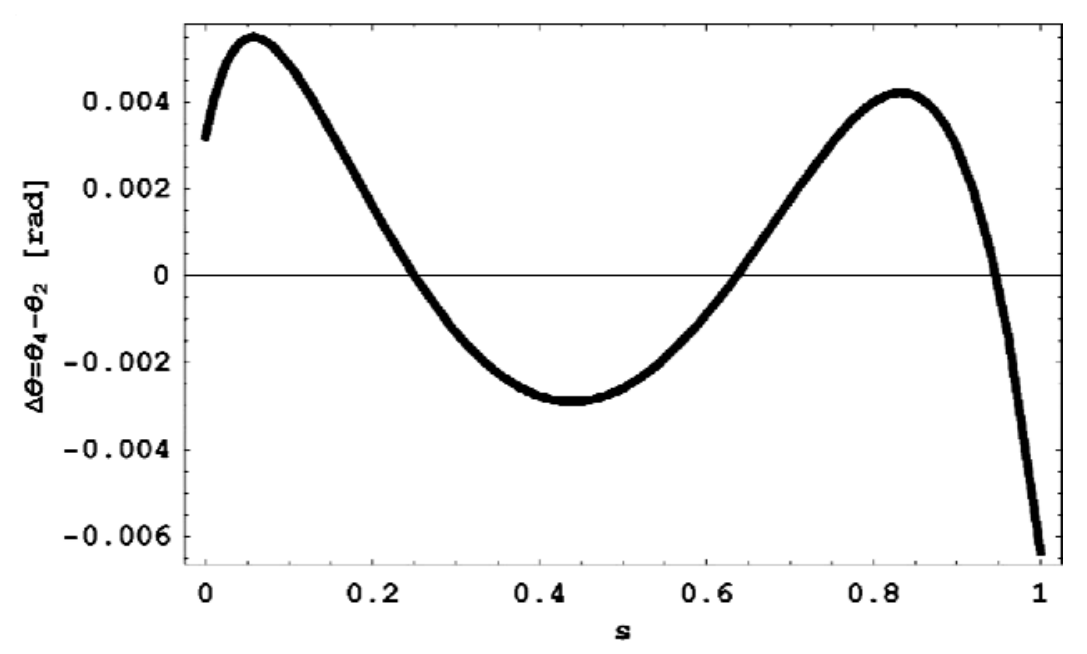

Fig.5. The relative angular motion of jaws when the load is transported.

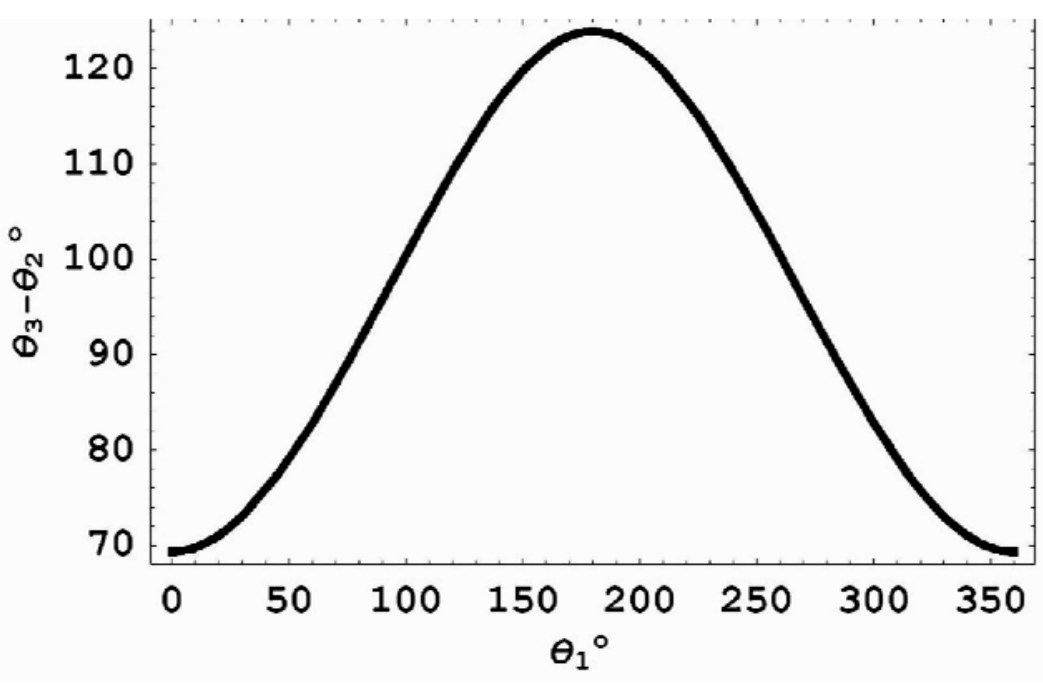

Fig.6. Transmission angle of the four bar linkage $\mathrm{O}_{1} \mathrm{ABO}_{2}$.

\section{Conclusions}

The synthesis method was adopted to design a simple, six-link kinematic feeder driven by one rotor. Compared with Buśkiewicz (2014), this paper presents a different approach in designing the feeder. The theoretical consideration is completed with a numerical solution. The results prove that the method can be applied to solve this specific task of mechanism synthesis. The illustrative example provides an insight into the size of the working spaces.

One can notice some disadvantages of the approach. The location of point $\mathrm{M}$ and the location of the ground pivot $\mathrm{O}_{3}$ are chosen manually. Moreover, the process of the design is not fully deterministic as the evolutionary algorithm is employed to search the four-bar linkage meeting the required geometric features. It follows that many simulations are to be carried out to find a satisfactory solution. For this reason the problem is open with respect to formulation of the mathematical formulae making it possible to find the optimal feeder dimensions. The studies should focus on making the method more automatic in 
searching for feeders optimally satisfying the prescribed requirements. As the paper deals only with the geometric synthesis, optimization of kinematic and dynamic features of the feeder is required.

\title{
Nomenclature
}

\author{
$E$ - objective function in the path synthesis problem \\ $l_{1}, l_{3}, l_{4}$ - lengths of the crank, rocker and immoveable link of a four-bar linkage \\ $l_{2}, l_{5}, l_{6}$ - lengths of coupler $\mathrm{AB}$ \\ $l_{7}, l_{8}-$ dimensions of coupler $\mathrm{MN}$ and output link $\mathrm{O}_{3} \mathrm{~N}$ \\ $x, y$ - coordinates of a point in the global system \\ $x^{\prime}, y^{\prime}$ - coordinates of a point in the local system attached to coupler AB \\ $x^{\prime \prime}, y^{\prime \prime}$ - coordinates of a point in the local system attached to coupler MN \\ $\beta-$ angle between arms $\mathrm{AD}$ and $\mathrm{DB}$ of coupler $\mathrm{AB}$ \\ $\theta_{i} \quad-$ angular position of $i$-th link \\ $\theta_{2 L} \quad$ - four-parameter angular position of coupler $\mathrm{AB}$ \\ $v_{1}, v_{2}, v_{3}, v_{4}$ - parameters of function $\theta_{2 L}$
}

\section{References}

Bulatovic R.R. and Dordevic S.R. (2009): On the optimum synthesis of a four-bar linkage using differential evolution and method of variable controlled deviations. - Mechanism and Machine Theory, vol.44, pp.235-246.

Buśkiewicz J. (2009): Minimization of the dimension of the solution space in optimal mechanism synthesis. - Publishing House of Poznan University of Technology, series Dissertations, No.442.

Buśkiewicz J. (2010): Use of shape invariants in optimal synthesis of geared five-bar linkage. - Mechanism and Machine Theory, vol.45, pp.273-290.

Buśkiewicz J. (2014): Design of a feeder with the use of the path synthesis method. - New Advances in Mechanisms, Transmissions and Applications Mechanisms and Machine Science, vol.17, pp.251-259.

Chao C. and Jorge A. (2008): A novel family of linkages for advanced motion synthesis. - Mechanism and Machine Theory, vol.43, pp.882-890.

Erdman A.G. and Sandor G.N. and Kota S.S. (2001): Mechanism Design: Analysis \& Synthesis. - 4th Ed. (Web Enhanced), vol.1, Prentice-Hall.

Gronowicz A., Jucha J., Miller S. and Twarog W. (1991): Mechanism and machine theory (in polish). - Wroclaw.

Hain K. (1967): Applied Mechanics 2nd ed.. - New York: McGraw-Hill Book Company.

Kramer and Sandor G.N. (1970): Kinematic Synthesis of Watt's Mechanism. - ASME Paper, No.70-mech-50.

Matekar S.B. and Gogate G.R. (2012): Optimum synthesis of path generating four-bar mechanisms using differential evolution and a modified error function. - Mechanism and Machine Theory, vol.52, pp.158-179.

McGarva J. and Mullineux G. (1994): Harmonic representation of closed curves. - Appl. Math. Model., vol.17, No.4, pp.213-218.

Patent, P.102470.

Patent P.125376.

Patent P.111933.

Penunuri F., Peón-Escalante R., Villanueva C. and Pech-Oy D. (2011): Synthesis of mechanisms for single and hybrid tasks using differential evolution. - Mechanism and Machine Theory, vol.46, No.10, pp.1335-1349. 
Radovan R., Bulatović R.R, Dordević S.R. and Dordević V.S. (2013): Search algorithm: A metaheuristic approach to solving the problem of optimum synthesis of a six-bar double dwell linkage. - Mechanism and Machine Theory, vol.61, pp.1-13.

Sanchez Marin F.T. and Gonzalez A.P. (2004): Open-path synthesis of linkages through geometrical adaptation. Mechanism and Machine Theory, vol.39, pp.943-955.

Suh C.H. and Radcliffe C.W. (1978): Kinematics and Mechanism Design. - New York: Wiley.

Ullah I. and Kota S. (1997): Optimal synthesis of mechanisms for path generation using Fourier descriptors and global search methods. - Journal of Mechanical Design, vol.119, pp.504-510.

Wen-Yi L. (2010): A GA-DE hybrid evolutionary algorithm for path synthesis of four-bar linkage. - Mechanism and Machine Theory, vol.45, No.8, pp.1096-1107.

Received: May 12, 2014

Revised: May 19, 2014 